\title{
Small-Scale Turbulence in a Closed-Field-Line Geometry
}

\author{
Paolo Ricci* and B. N. Rogers ${ }^{\dagger}$ \\ Department of Physics and Astronomy, Dartmouth College, Hanover, New Hampshire 03755, USA
}

\section{W. Dorland}

Department of Physics, University of Maryland, College Park, Maryland 20742, USA

(Received 30 May 2006; published 15 December 2006)

\begin{abstract}
Plasma turbulence due to small-scale entropy modes is studied with gyrokinetic simulations in a simple closed-field-line geometry, the $Z$ pinch, in low- $\beta$ parameter regimes that are stable to ideal interchange modes. We find an enormous variation in the nonlinear dynamics and particle transport as a function of two main parameters, the density gradient and the plasma collisionality. This variation is explained in part by the damping and stability properties of spontaneously formed zonal flows in the system. As in toroidal systems, the zonal flows can lead to a strong nonlinear suppression of transport below a critical gradient that is determined by the stability of the zonal flows.
\end{abstract}

DOI: 10.1103/PhysRevLett.97.245001

PACS numbers: 52.35.Ra, 52.30.Gz, 52.58.Lq

We address the transport of particles induced by smallscale turbulence in a simple closed-field-line geometry, the $Z$ pinch, in which a cylindrically symmetric plasma is confined by a poloidal magnetic field $B_{\theta}(r)[1]$. We find that the physics of this turbulence is similar in many ways to that found in widely studied toroidal systems (see, e.g., Ref. [2], and references therein). It is generated by highly anisotropic $\left(k_{\perp} \gg k_{\|}\right)$nonmagnetohydrodynamic (MHD) instabilities at the ion-gyroradius scale, which are driven by a combination of the pressure gradient and magnetic curvature and have growth rates comparable to the diamagnetic and curvature drift frequencies. Nonlinearly, the breakup of these modes generates zonal flows that, below a critical gradient, act to strongly suppress the transport. The magnitude of this critical gradient, also as in the toroidal case, is determined by the stability properties of the zonal flows [2]. Thus, the $Z$-pinch system, in addition to its intrinsic interest, offers an analytically and numerically simple test bed in which to explore physics that underlies transport in more complex geometries.

We consider the regime of plasma $\beta \ll 1$, in which the dominant instabilities take on an electrostatic character and have $k_{\|}=0$ [3]. At sufficiently steep gradients, the fastest growing mode is a collisionless ideal MHD interchange instability with $\gamma_{\text {ideal }}^{2}=\left(c_{s}^{2} / R\right)\left[2 / L_{p}-7 / R\right]\left[c_{s}^{2}=\left(T_{e}+\right.\right.$ $\left.T_{i}\right) / m_{i}, R$ is the curvature radius at $r=R$, and $L_{p}=$ $-p / p^{\prime}$ an equilibrium pressure-gradient scale length, with $p^{\prime}=d p / d r$ ] [4]. At weaker gradients $\left(L_{p} / R>\right.$ $2 / 7 \simeq 0.29$ ), the ideal mode is stable, leaving behind a shorter-wavelength non-MHD mode known as the entropy mode [3-6]. In a collisionless plasma with $T_{e}=T_{i}$ and no temperature gradients, the entropy mode is unstable for $2 / 7<L_{n} / R<\alpha$, where $\alpha$ depends on the physics model: In the gyrokinetic model used in this Letter, $\alpha=\pi / 2$ [4]. Simple two-fluid models predict a lower threshold $\alpha \sim$ 0.7. Figure 1 shows the growth rate of the entropy mode when $T_{i}=T_{e}, \nu=0$, and $T^{\prime}=0$, obtained from the gyrokinetic model as a function of $k_{z} \rho_{s}$ for various values of $L_{n} / R$, where $k_{z}$ is the wave number along the axis of the plasma column and $\rho_{s}=c_{s} / \Omega_{c i}$. It is seen that $\gamma$ increases linearly with $k_{z}$ up to roughly $k_{z} \rho_{s} \sim 1$, where the largest growth rates are attained. At steeper gradients, these growth rates become comparable to those of the ideal interchange mode.

Here we explore the turbulence and nonlinear transport arising from the entropy mode in ideally stable parameter regimes for systems in which $L_{n}$ and $R$ are both much larger than $\rho_{s}$. To address the regime of $T_{i} \gtrsim T_{e}$, a kinetic model valid to all orders in $k_{\perp} \rho_{i}\left(\sim k_{\perp} \rho_{s}\right)$ is clearly required. We therefore use here the GS2 code $[7,8]$ to solve the nonlinear gyrokinetic equations for both ions and electrons (see, e.g., [9]). The simulations are carried out in the

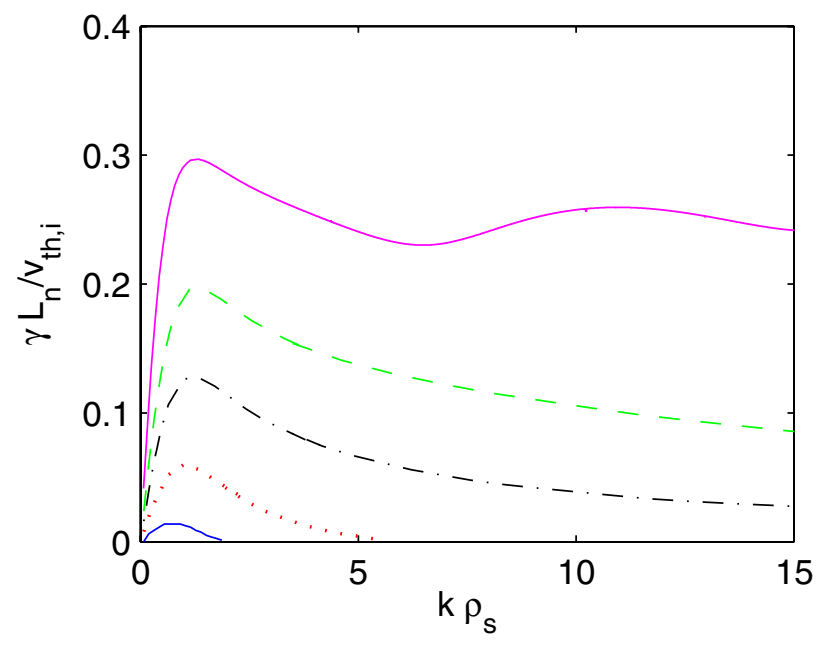

FIG. 1 (color online). $\quad \gamma$ vs $k \rho_{s}$ for (bottom to top): $L_{n} / R=$ 1.25 (solid blue curve), $L_{n} / R=1$ (dotted red curve), $L_{n} / R=$ 0.8 (dotted-dashed black curve), $L_{n} / R=0.67$ (dashed green curve), and $L_{n} / R=0.5$ (solid magenta curve). 
flux-tube approximation [10], and periodic boundary conditions for the turbulent perturbations are imposed in all directions. Collisions are modeled by a gyro-averaged Lorentz collision operator described in Ref. [4]. This operator accounts for finite Larmor radius effects that are the main source of collisional damping in our system. It conserves particle number and energy but not momentum. A more refined collision operator is currently under development.

We focus on the impact of two main parameters: the plasma collisionality and $L_{n} / R$. The other parameters in our simulations, unless otherwise noted, are $T_{i}=T_{e}, T_{i}^{\prime}=$ $T_{e}^{\prime}=0, \beta \ll 1$, and $m_{i} / m_{e}=1836$. The collision frequency is defined as $\nu=\pi n_{0} e^{4} \ln \Lambda /\left(T_{i}^{3 / 2} m_{i}^{1 / 2}\right)$, and it is normalized to $v_{\mathrm{th}, i} / R$. As one might expect given the $k_{\|}=$ 0 nature of the dominant linear modes for $\beta \ll 1$, nonlinear simulations performed in three spatial dimensions show that the dynamics of the system remain nearly uniform along the equilibrium magnetic field. Thus, the simulations described here are carried out mainly in the twodimensional $(r, z)$ plane transverse to the magnetic field. We have performed convergence studies of the nonlinear transport for all of the simulations presented here, varying the number of spatial Fourier modes, the size of the spatial domain, the simulation-box aspect ratio (by a factor of 4), and the (2D) energy grid. The largest simulations typically include Fourier modes in the range $0.05 \leq k_{x} \rho_{i}, k_{y} \rho_{i} \leq 5$, 24 grid points in the particle (ion or electron) energies in the velocity range $0 \leq v \leq 6 v_{\text {th }}$, and 10 points in $\xi=$ $v_{\|} / v$. We have also included in the simulations an artificial hyperviscous term in the gyrokinetic equation that acts equally on the full guiding center ion and electron distribution functions and leads to dissipation proportional to $k^{4}$. Based on convergence tests such as that shown in Table I, we find that the transport is not sensitive to the level of this hyperviscosity provided that it is in a certain range. If the hyperviscosity is smaller than a threshold value, however, the asymptotic level of the transport, after coming to a quasistationary value, eventually starts to grow linearly with time on a very long time scale (thousands of $\left.R / v_{\text {th }, i}\right)$. This growth becomes progressively weaker as the spatial resolution is improved and seems to arise from the lack of damping in the system at small scalesdamping that, in a physical system, would presumably be present at sufficiently high $k$.

In the typical setup of the runs, initial low-amplitude random noise leads to the growth of a spectrum of unstable

TABLE I. Hyperviscosity study for $L_{n} / R=1, \nu=0.01$. The hyperviscosity term in the gyrokinetic equations is given by $\nu_{\text {hyp }}\left(k / k_{\max }\right)^{4}$, where $k_{\max } \rho_{i}=2.5$ for the present simulations and $\nu_{\text {hyp }}$ is normalized to $v_{\text {th }, i} / R$.

\begin{tabular}{lcccc}
\hline \hline$\nu_{\text {hyp }}$ & 0.01 & 0.1 & 1 & 10 \\
\hline$\Gamma_{\text {part }}^{\infty}$ & 0.0082 & 0.0062 & 0.0087 & 0.0269 \\
\hline \hline
\end{tabular}

entropy modes. As the electric potential $\phi$ in Fig. 2(a) illustrates, the most unstable modes with $k_{z} \rho_{s} \sim 1$ and $k_{r}=0$ eventually dominate in the late linear phase, producing $\mathbf{E} \times \mathbf{B}$ plasma flows in the radial direction. These flows produce radial particle transport $\Gamma_{\text {part }}=\left\langle n v_{E, r}\right\rangle$ (where $\mathbf{v}_{E}$ is the gyro-averaged $\mathbf{E} \times \mathbf{B}$ drift velocity and $\langle\ldots\rangle$ denotes the spatial average over the simulation domain) that grows exponentially with the instability until $t \sim 220$ [see Fig. 2(c); here $t$ is normalized to $R / v_{\text {th }, i}$ and $\Gamma_{\text {part }}$ to $\left.\left(\rho_{i} / R\right)^{2} n_{0} v_{\mathrm{th}, i}\right]$. At this point, the growth of the primary (entropy) modes triggers the onset of a conventional Kelvin-Helmholtz instability (KHI) with finite $k_{r} \rho_{i} \sim 0.5$ [see Fig. 2(b)]. The nonlinear development of the KHI leads to a strong burst of sheared $E \times B$ flows in the $z$ direction (zonal flows) which saturate the primary and quickly reduce the particle flux to a significantly lower level. Following this, the particle flux fluctuates about a stationary value $\Gamma_{\text {part }}^{\infty}$. The frequency of the fluctuations at a given gradient is typically comparable to the peak linear growth rate. Further tests confirm that $\Gamma_{\text {part }}^{\infty}$ does not depend on the initial conditions: Simulations started from large or small amplitude perturbations of various types, for example, yield essentially the same asymptotic fluxes. A possible exception is the collisionless case at weak gradients, when the transport becomes negligibly small $\left(\Gamma_{\text {part }} \lesssim 10^{-5}\right)$.

In Fig. 3(a), we plot $\Gamma_{\text {part }}^{\infty}$ as a function of $L_{n} / R$ and $\nu$. At gradients comparable to or somewhat steeper than the fluid model entropy-mode stability boundary $\left(L_{n} / R \sim 0.6\right)$, the particle flux reaches robust, gyro-Bohm levels $\Gamma_{\text {part }}^{\infty} \gtrsim 1$ and becomes insensitive to the plasma collisionality. At only modestly weaker gradients, on the other hand, the transport is smaller by 2 orders of magnitude or more and exhibits a strong dependence on $\nu$. This strong $\nu$ dependence at weak gradients is due to the collisional damping
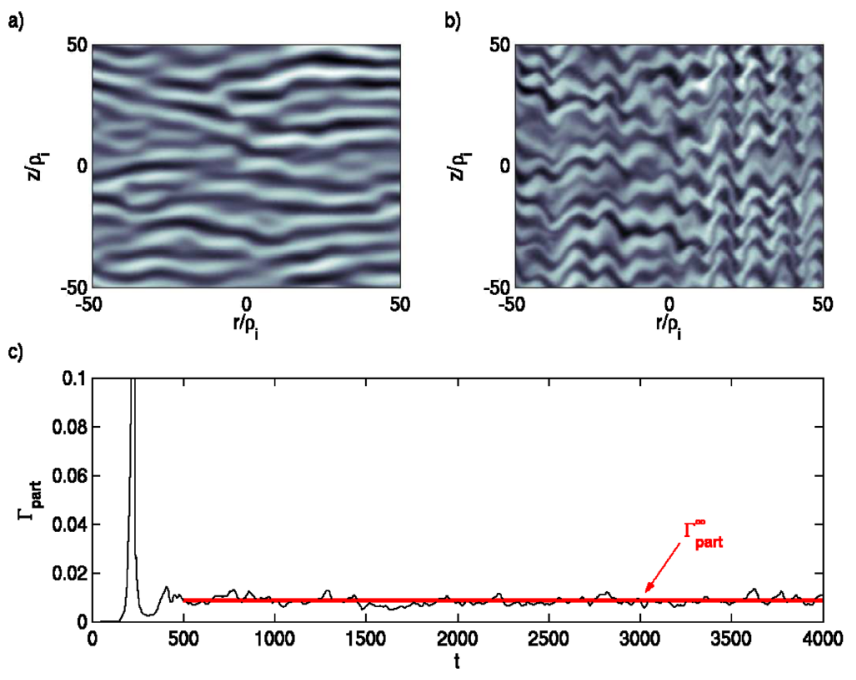

FIG. 2 (color online). $\phi$ at (a) $t=207$ and (b) $t=230$. (c) shows $\Gamma_{\text {part }}$ for $L_{n} / R=1, \nu=0.01$. 

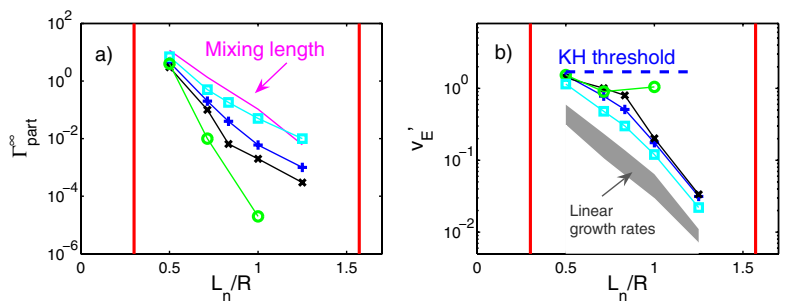

FIG. 3 (color online). (a) $\Gamma_{\text {part }}^{\infty}$ vs $L_{n} / R$; (b) zonal-flow shearing rates $V^{\prime}$. Light-blue squares, $\nu=0.1$; dark blue,$+ \nu=0.01$; black $\times, \nu=0.001$; green circles, $\nu=0$. The vertical lines delimit the entropy-mode instability region.

of zonal flows in the system. As can be seen from the snapshot of $\phi$ in Fig. 4(a) for $L_{n} / R=1$ and $\nu=0$, the simulations in the low collisionality, low gradient regime at late times are dominated by quasistatic zonal flows with $k_{z}=0$ and finite $k_{r}$ (vertical stripes). These zonal flows are robust and arise regardless of the initial conditions of the simulations. If a turbulent simulation is restarted with the zonal flows initially reset to zero, for example, the flows come back on a time scale $t \sim 1 / \gamma$. These zonal-flow modes are generated by the nonlinear evolution of the KHI and are associated with a huge reduction in the transport. At the highest $\nu$ values in Fig. 3(a) $(\nu=0.1$, squares), for example, the zonal flows are strongly damped and the transport is much larger. Similarly, if the collisionless simulations (circles) are carried out with the zonal flows artificially kept at very low levels, the transport becomes comparable to those of the largest- $\nu$ runs. A snapshot of the turbulence in the intermediate collisional case (e.g., $\nu=0.01$ ) is shown in Fig. 4(b). Zonal flows are still present in the simulation, but their collisional decay leads to the reemergence of the primary (entropy) modes that increase the level of particle transport. The wave
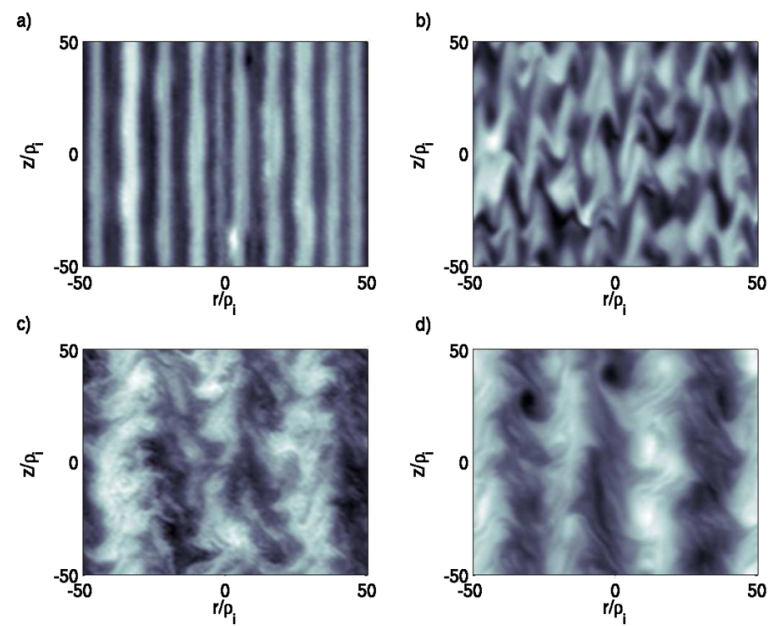

d)

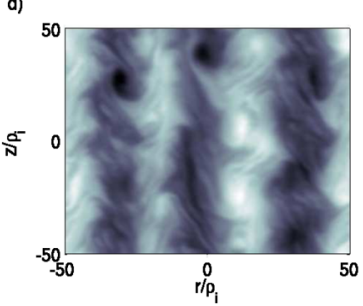

FIG. 4 (color online). $\phi \quad$ at $\quad$ (a) $L_{n} / R=1, \quad \nu=0$; (b) $L_{n} / R=1, \nu=0.01$; (c) $L_{n} / R=0.5, \nu=0$; and $L_{n} / R=$ $0.5, \nu=0.01$. numbers of the dominant zonal flows range from $k_{r} \rho_{i} \sim$ 0.2 at $L_{n} / R=0.5$ to $k_{r} \rho_{i} \sim 0.5$ at $L_{n} / R=1.25$.

In the higher- $\nu$ runs, the absence of strong, dominating zonal flows in the system allows the transport to be wellestimated by a mixing-length argument. Since $d / d t=$ $\partial / \partial t+\mathbf{v}_{E} \cdot \nabla \sim \gamma+k_{r} k_{z} \phi$, one would expect nonlinear effects to become important when $\phi \sim \gamma /\left(k_{r} k_{z}\right) \sim \gamma / k^{2}$ [here $k$ is normalized to $1 / \rho_{i}$ and $\phi$ to $\left.T_{i} \rho_{i} /(e R)\right]$. From the continuity equation for the density, and as numerical results confirm, in the turbulent state $\phi \sim n$, where $n$ is the density perturbation (normalized to $n_{0} \rho_{i} / R$ ). Thus, $\Gamma_{\text {part }} \sim$ $k\langle n \phi\rangle \sim \gamma^{2} / k^{3}$. Regarding the choice of $k$, the simulations show that the transport is driven predominantly by modes with $k \rho_{i} \sim 0.2$ across the range of parameters considered here. With this value of $k$, the mixing-length estimate of the transport is plotted in Fig. 3(a). It is seen that the scaling $\Gamma_{\text {part }}^{\infty} \propto \gamma^{2}$ is reasonably accurate at the higher- $\nu$ values, suggesting that the weakness of the transport at weaker gradients (where the mode is unstable only in the kinetic limit) is due in large part to the smallness of the linear growth rates in the kinetic regime. The further suppression of the transport below such values arising for smaller $\nu$ is due to the zonal flows.

Figure 3(b) shows the spatial root mean square shearing rate due to the zonal flows, $V^{\prime}=d v_{E, z} / d r$, evaluated during the turbulent phase of the simulations (normalized to $\left.R / v_{\mathrm{th}, i}\right)$ and averaged over time. Consistent with the mixing-length argument, in the most collisional cases or even in the collisionless simulation at the highest gradient $\left(L_{n} / R=0.5\right)$, the shearing rates are comparable to the linear growth rates in the absence of the zonal flows (gray band). We have recalculated the linear growth rates in the various cases in the presence of the zonal flows by restarting the simulations in the asymptotic regime with all modes other than the zonal flows artificially reduced to very small levels. These simulations show that, in the most extreme cases, the entropy-mode growth rates are greatly reduced, but not eliminated, by the velocity shear. At $\nu=$ $0, L_{n} / R=1$ (in which $V^{\prime}$ is about 10 times larger than the largest growth rates in the unsheared system), for example, the zonal flows reduce linear growth rates by about a factor of 5 or more. Significantly, the most unstable of these residual (unquenched) linear modes are localized radially to regions of weak velocity shear (i.e., nodes of $\phi$ ). In the weak gradient cases, they saturate at a very low level and have little impact on the zonal flows.

Given the absence of magnetic shear in the Z-pinch system, one might wonder why the zonal flows seen in Fig. 4(a), for example, are not unstable to the KHI. There are two stabilizing factors: the ion diamagnetic drift associated with the equilibrium density gradient $L_{n}$ and the magnetic curvature. In the absence of magnetic curvature and background gradients, our tests show that the zonal flows in the simulation with $L_{n} / R=1, \nu=0$ [Fig. 4(a)] are indeed unstable to a fast growing KHI with a linear 

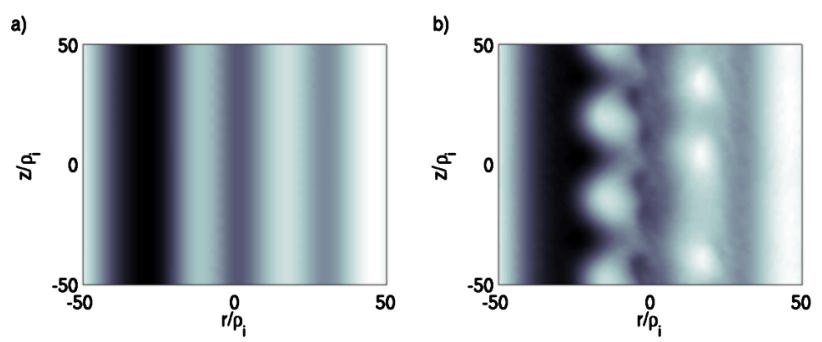

FIG. 5 (color online). $\quad \phi$ at $L_{n} / R=0.5$ and $\nu=0.01$ showing the onset of the KHI (b) when the zonal flows are artificially doubled upon restart.

growth rate $\gamma \sim 0.2$ at $k_{z} \rho_{i} \sim 0.5$. Consistent with the findings of Ref. [11], if the background density gradient alone is added back into the system, this peak KHI growth rate is reduced to about $\gamma \sim 0.13$. Finally, if the magnetic curvature is also included, the KHI becomes stable for the flow amplitudes present in the simulation. This stabilization arises fundamentally from finite plasma compressibility and is discussed in more detail below. At levels of $V^{\prime}$ that are slightly higher than the maximum values observed in the simulations, however, the zonal flows do eventually become unstable to the KHI. This was determined by restarting the late-time simulations at various values of $L_{n} / R$ with the zonal flows increased to artificially higher levels and observing the fate of the zonal flows. An example is shown in Fig. 5 in the case of $L_{n} / R=0.5$, which shows the onset of the KHI with a linear growth rate of $\gamma \sim$ 0.5 that was triggered by a doubling of the zonal flows upon restart. Over the range of $L_{n} / R$ shown in Fig. 3(b), the KHI threshold is roughly flat and is bracketed by $V^{\prime}=$ $1.5 \pm 0.4$, as shown in the figure.

In the threshold regime for zonal-flow breakup, the KHI growth rate is comparable to the background diamagnetic and curvature drift frequencies, and its detailed analytic study is beyond the scope of this Letter. It is not difficult to show, however, that higher frequency modes (i.e., those for which $\omega_{*}$ and $\omega_{d}$ effects are negligible) also exhibit a stability threshold due to curvature and compressibility. Assuming $d / d t \gg \omega_{*}, \omega_{d}$ and $k_{\perp} \rho_{i} \ll 1$, the KHI in our system is governed by the eigenvalue equation

$$
\frac{d}{d r}\left(\bar{\gamma}^{2} \frac{d f}{d r}\right)=k_{z}^{2}\left(\bar{\gamma}^{2}-\gamma_{\text {ideal }}^{2}\right) f,
$$

where $\bar{\gamma}(r)=\gamma+i k_{z} V(r), f=\tilde{\phi} / \bar{\gamma}, \tilde{\phi}$ is the perturbation in the electrostatic potential associated with the KH eigenmode, and $\gamma_{\text {ideal }}$ (the ideal interchange growth rate) was given earlier. When curvature is neglected (so that $\gamma_{\text {ideal }}=0$ ), this equation describes standard $\mathrm{KH}$ modes, while the limit $V=0$ leads to (radially constant) local ideal interchange solutions with $\gamma^{2}=\gamma_{\text {ideal }}^{2}$. In the parameter regime of our simulations, due to finite plasma compressibility, $\gamma_{\text {ideal }}^{2}<0$, and, thus, the coupling of the system to the ideal interchange mode is stabilizing. This stabilizing contribution leads to a threshold in $V^{\prime}$ $\left(\sim\left|\gamma_{\text {ideal }}\right|\right)$ below which the KHI is stable $\left(\simeq 1.8\left|\gamma_{\text {ideal }}\right|\right.$ for a sinusoidal sheared flow).

The presence of the KHI threshold explains why the transport is sensitive to $\nu$ at large $L_{n} / R$ but not at $L_{n} / R \simeq$ 0.5 . At large $L_{n} / R$, the amplitudes of the zonal flows in the collisional cases are regulated by collisional damping and are well below the KHI threshold. As $L_{n} / R$ is decreased, however, the growth rates of the primary (entropy) modes become stronger, and the zonal flows (generated by the KH secondary) become stronger as well, until finally, at $L_{n} / R \simeq 0.6$, the shearing rates reach the threshold for tertiary KHI, even at the strongest values of $\nu$. At this point, the amplitudes of the flows become controlled by the KHI, and collisional damping of the flows becomes unimportant. This insensitivity to $\nu$ at $L_{n} / R=0.5$ is also reflected by the profiles of $\phi$ shown in Figs. 4(c) $(\nu=0)$ and $4(\mathrm{~d})(\nu=0.01)$.

We therefore conclude that, although the nature of the tertiary instability in the Z-pinch system (the KHI) differs from that prevailing in toroidal systems $[2,12]$, one important characteristic remains similar: When collisions are sufficiently weak, anomalously small transport levels (far below mixing-length estimates) arise at gradients that are sufficiently close to marginal linear stability (here $L_{n} / R \geqslant$ $0.6)$, where the growth rates of the primary modes are well below the instability limit for zonal-flow breakup.

Financial support from NASA Sun-Earth Connection Theory Program under Grant No. NNG05GJ70G and from the DOE Center for Multiscale Plasma Dynamics are acknowledged.

*Electronic address: paolo.ricci@dartmouth.edu †Electronic address: barrett.rogers@dartmouth.edu *Electronic address: bdorland@umd.edu

[1] J.P. Freidberg, Ideal Magnetohydrodynamics (Plenum, New York, 1987).

[2] B. N. Rogers et al., Phys. Rev. Lett. 85, 5336 (2000).

[3] A. N. Simakov et al., Phys. Plasmas 8, 4414 (2001).

[4] P. Ricci et al., Phys. Plasmas 13, 062102 (2006).

[5] J. Kesner, Phys. Plasmas 7, 3837 (2000).

[6] J. Kesner and R. J. Hastie, Phys. Plasmas 9, 395 (2002).

[7] M. Kotschenreuther et al., Comput. Phys. Commun. 88, 128 (1995).

[8] W. Dorland et al., Phys. Rev. Lett. 85, 5579 (2000).

[9] E. A. Frieman and L. Chen, Phys. Fluids 25, 502 (1982).

[10] M. A. Beer et al., Phys. Plasmas 2, 2687 (1995).

[11] B. N. Rogers and W. Dorland, Phys. Plasmas 12, 062511 (2005).

[12] A. M. Dimits et al., Phys. Plasmas 7, 969 (2000). 